\title{
Spectral Curves and the ADHM Method
}

\author{
N. J. Hitchin ${ }^{1}$ and M. K. Murray \\ ${ }^{1}$ Mathematical Institute, 24-29 St. Giles, Oxford. OX13LB, United Kingdom \\ ${ }^{2}$ School of Mathematical Sciences, The Flinders University of South Australia, Bedford Park. SA \\ 5042, Australia
}

\begin{abstract}
For a general monopole the algebraic curves defined by Nahm are shown to be the same as the spectral curves.
\end{abstract}

\section{Introduction}

It is shown in [6] that an $S U(n)$ monopole or solution of the Bogomolny equations with maximal symmetry breaking at infinity has associated to it a collection of $n-1$ algebraic curves $S_{1}, \ldots, S_{n-1}$ whose intersections $S_{i} \cap S_{i+1}$ decompose as $S_{i+1, i}+S_{i-1, i}$. Following [3] these are called spectral curves and a general monopole is completely determined by these curves and the splitting of $S_{i} \cap S_{i+1}[6]$.

Nahm, using his adaption of the Atiyah-Drinfeld-Hitchin-Manin (ADHM) approach to instantons, has shown how to associate to a monopole a possibly different set of $n-1$ curves. The purpose of this paper is to show that the spectral curves and the Nahm curves always coincide for a general monopole. It provides in the particular case of $S U(2)$ a replacement for [4] Sect. 7, which is incorrect due to a sign error.

In Sect. 2 some basic facts and the construction of Nahm's spectral curve is reviewed. Each of Nahm's curves is constructed from a vector space $W_{z}$ and three endomorphisms $T_{i}(z) \in \operatorname{End}\left(W_{z}\right), i=1,2,3$.

Section 3 shows how to realize the spectral curves by applying Nahm's methods to a different vector space $V_{z}$ and endomorphisms $H_{i}(z) \in \operatorname{End}\left(V_{z}\right)$. In the final section an isomorphism is constructed from $V_{z}$ to $W_{z}$ which intertwines $H_{i}(z)$ and $T_{i}(z)$ for $i=1,2,3$, and thereby proves the identity of the curves. This isomorphism is provided by the Penrose correspondence between solutions to zero rest mass field equations and elements of sheaf cohomology groups, reduced to the three-dimensional situation. It is the link between the algebraic geometry of the spectral curve and the analytical origin of the Nahm curve.

As this paper relies heavily on the methods of [4] we shall, in the interests of brevity, assume that the reader has a copy of it near at hand. Throughout we shall adopt the same notation for a holomorphic bundle and its sheaf of sections, in particular $\mathcal{O}(k)$ will denote the holomorphic line bundle one $\mathbf{P}_{1}$ of Chern class $k$ or its pullback to $T \mathbf{P}_{1}$. 


\section{Review}

We review the notation and results for an $S U(n)$ monopole $(A, \Phi)$ with maximal symmetry breaking at infinity [6]. The asymptotic expansion of the Higgs field can be assumed to be

$$
\Phi(0,0, t)=i\left[\begin{array}{ccc}
\mu_{1} & \cdots & 0 \\
\vdots & \ddots & \vdots \\
0 & \cdots & \mu_{n}
\end{array}\right]-\frac{i}{2 t}\left[\begin{array}{ccc}
k_{1} & \cdots & 0 \\
\vdots & \ddots & \vdots \\
0 & \cdots & k_{n}
\end{array}\right]+o\left(\frac{1}{t^{2}}\right)
$$

where $\sum_{i=1}^{n} \mu_{i}=\sum_{i=1}^{n} k_{i}=0$ and $\mu_{1}>\cdots>\mu_{n}$. The magnetic charges of the monopole are defined by $m_{1}=k_{1}, \ldots, m_{n-1}=k_{1}+\cdots+k_{n-1}$ and are all non-negative.

When $(A, \Phi)$ satisfies the Bogomolny equations and appropriate boundary conditions [6] it defines a holomorphic bundle $E$ of rank $n$ on the space $\mathscr{T}=T \mathbf{P}_{1}$ of all oriented lines in $\mathbf{R}^{3}$. The fibre over a line $\gamma$ is the vector space of all functions $s: \gamma \rightarrow \mathbf{C}^{n}$ satisfying

$$
\left(\nabla_{\gamma}-i \Phi\right) s=0,
$$

where $\nabla_{\gamma}$ is the covariant derivative defined by $A$ restricted to the line. The collection of all lines through $x \in \mathbf{R}^{3}$ defines a $\mathbf{P}_{1} \subset \mathscr{T}$, the real section determined by $x$, and $E$ is holomorphically trivial on real sections. Substituting (1) into (2) and studying the asymptotic decay of solutions in the $+\infty$ and $-\infty$ directions along the line defines two families of holomorphic sub-bundles,

$$
\begin{aligned}
& E_{1}^{+} \subset E_{2}^{+} \subset \cdots \subset E_{n}^{+}=E \\
& E_{1}^{-} \subset E_{2}^{-} \subset \cdots \subset E_{n}^{-}=E,
\end{aligned}
$$

with quotients

and

$$
E_{i}^{+} / E_{i-1}^{+}=L^{\mu_{1}}\left(-k_{i}\right) \quad i=2, \ldots, n,
$$

$$
E_{i+1}^{-} / E_{i}^{-}=L^{\mu_{n-1}}\left(k_{n-1}\right) \quad i=1,2, \ldots, n-1 .
$$

The line bundle $L^{\mu}$ is constructed from a $U(1)$ monopole with $A=0, \Phi=i \mu$ and is defined in [3]: $L^{\mu}(k)$ is $L^{\mu}$ tensored with the pullback from $\mathbf{P}_{1}$ of the holomorphic line bundle with Chern class $k$.

The $p^{\text {th }}$ spectral curve $S_{p}$ is defined to be the set of $\gamma$, where $E_{p}^{+} \cap E_{n-p}^{-} \neq \emptyset$ or as the divisor $\mathscr{T}$ determined by the zeros of

$$
\varphi_{p}: \Lambda^{p} E_{p}^{+} \rightarrow \Lambda^{p}\left(E / E_{n-p}^{-}\right) .
$$

It is worth noting that if we work with $\Phi_{z}=\Phi-i z, z \in \mathbf{R}$ as Nahm does [8], then for each $z$ we can consider the set $S_{z}$ where (2) has integrable solutions and then

and

$$
S_{z}=S_{p} \quad z \in\left(\mu_{p-1}, \mu_{p}\right)
$$

$$
S_{z}=\emptyset \quad \text { otherwise. }
$$

Nahm's method of defining algebraic curves associated to a monopole is as follows [8]. 
For each $z$ solve the Dirac equation for spinors coupled to $\mathbf{C}^{n}$, that is,

$$
\left(D_{A}+\Phi_{z}\right) \varphi=0
$$

where $D_{A}$ is the covariant Dirac operator. Using an index theorem and vanishing theorem Nahm calculates that $W_{z}$, the space of integrable solutions to (5), satisfies

$$
\begin{aligned}
W_{z} & =0, \quad z<\mu_{n}, \quad \mu_{1}<z, \\
\operatorname{dim} W_{z} & =m_{p}, \quad z \in\left(\mu_{p+1}, \mu_{p}\right) .
\end{aligned}
$$

This defines a vector bundle $W$ on each $\left(\mu_{p+1}, \mu_{p}\right)$ which is a sub-bundle of the trivial bundle $L^{2}\left(\mathbf{R}^{3}, \mathbf{C}^{2} \otimes \mathbf{C}^{n}\right) \times\left(\mu_{p+1}, \mu_{p}\right)$. If $\pi$ is the orthogonal projection onto $W$, then Nahm defines three endomorphisms and a connection on $W$ by

$$
\begin{aligned}
& T_{j}(\varphi)=i \pi\left(x_{j} \varphi\right), \quad j=1,2,3, \\
& \nabla_{z}(\varphi)=\pi\left(\frac{\partial \varphi}{\partial z}\right) .
\end{aligned}
$$

These satisfy Nahm's equations

$$
\nabla_{z} T_{1}=\left[T_{2}, T_{3}\right]
$$

and all cyclic permutations.

Now consider $\mathbf{P}_{1}$ embedded as a conic in $\mathbf{P}_{2}$ and let $z_{1}, z_{2}, z_{3}$ be the sections of $\mathcal{O}(2)$ over $\mathbf{P}_{1}$ induced by the co-ordinates on $\mathbf{P}_{2}$. In terms of the co-ordinates used in [3],

$$
z_{1}=-i\left(1+\zeta^{2}\right), \quad z_{2}=1-\zeta^{2}, \quad z_{2}=-2 \zeta .
$$

Denote also by $z_{i}$ the pullback of these to $\mathscr{T}$ and note that because $\mathscr{T} \simeq \mathcal{O}(2)$ the bundle $\mathcal{O}(2)$ pulled back to $\mathscr{T}$ has a tautological section $\eta$. In fact in [3] it is shown that $\eta, z_{1}, z_{2}, z_{3}$ form a basis of $H^{0}(\mathscr{T}, \mathcal{O}(2))$. Now using the map $\operatorname{det}: \operatorname{End}\left(W_{z}\right) \rightarrow \mathbf{C}$ we can define a section of $\mathcal{O}\left(2 m_{i}\right)$ on $\mathscr{T}$ by

$$
\operatorname{det}\left(\eta \cdot 1+\sum_{j=1}^{3} i z_{j} T_{j}(z)\right)
$$

if $z \in\left(\mu_{p+1}, \mu_{p}\right)$. The divisor of this section is, in fact independent of $z \in\left(\mu_{p+1}, \mu_{p}\right)$ (see $[8$ or 4$]$ ) and determines a curve $N_{p}$. We shall show that $N_{p}=S_{p}$ the $p^{\text {th }}$ spectral curve.

The method of proof is to produce a space $V_{z}$ isomorphic to $W_{z}$ and endomorphisms $H_{i}(z)$ which are equal to $T_{i}(z)$ under the isomorphism. Because we shall show that the above construction applied to $V_{z}$ and $H_{i}(z)$ gives $S_{p}$ the result will follow.

\section{The Twistor Viewpoint}

In this section we construct using twistor methods the bundle $V_{z}$ and the endomorphisms $H_{i}(z)$ and show that the divisor of

$$
\operatorname{det}\left(\eta \cdot 1+i \sum_{j=1}^{3} z_{j} H_{j}(z)\right)
$$

is the spectral curve $S_{p}$ if $z \in\left(\mu_{p+1}, \mu_{p}\right)$. 
The space used for $V_{z}$ in the $S U(2)$ case [4] was $H^{0}\left(S, L^{-z}\left(E_{1}^{+} \cap E_{1}^{-}\right)\left(2 m_{1}-1\right)\right)$ and this suggests that we look at

$$
H^{0}\left(S_{p}, L^{-z} I_{p}\left(2 m_{p}-1\right)\right)
$$

where $I_{p}=E_{p}^{+} \cap E_{n-p}^{-}$is the $p^{\text {th }}$ intersection bundle (more correctly a sheaf with support on $S_{p}$ ). To make $I_{p}$ behave reasonably we must look only at general monopoles. Recall that $S_{i} \cap S_{i+1}$ splits as $S_{i, i+1} \cup S_{i+1, i}$ [7], then we have

Definition 12. A monopole is general if

(1) The $S_{i, i+1}$ and $S_{i+1, i}$ are sets of distinct points without multiplicity, and

(2) The $S_{i, i+1}$ and $S_{i+1, i}$ do not intersect the singular set of the spectral curves.

Any $S U(2)$ monopole is general, and it was shown in [7] that general monopoles form a non-empty open set in the space of all monopoles.

Now we also have

Lemma 13. The spectral curve of a general monopole has no multiple components.

Proof. If the curve $S_{p}$ has a multiple component, then we must have $S_{p-1}=\phi$ and $S_{p+1}=\phi$, and then the proof in [4] can be applied.

Using this definition we have

Proposition 14. For a general monopole $\operatorname{dim}\left(I_{p}\right)=1$ at all points of $S_{p}$.

Proof. If we examine the definition of the $S_{i j}$ and the Bruhat decomposition of the flag manifold, we see that the points of $S_{p, p+1}, S_{p+1, p}, S_{p-1, p}$ and $S_{p, p-1}$ are characterised as follows:

$$
\begin{aligned}
& S_{p-1, p}^{-}: \operatorname{dim} E_{p-1}^{+} \cap E_{n-p}^{-} \geqq 1, \quad S_{p, p-1}: \operatorname{dim} E_{p}^{+} \cap E_{n-p+1}^{-} \geqq 2, \\
& S_{p, p+1}: \operatorname{dim} E_{p}^{+} \cap E_{n-p-1}^{-} \geqq 1, \quad S_{p+1, p}: \operatorname{dim} E_{p+1}^{+} \cap E_{n-p}^{-} \geqq 2 .
\end{aligned}
$$

It follows that if $\operatorname{dim} E_{p}^{+} \cap E_{n-p}^{-} \geqq 2$ at some point on $S_{p}$, then that point is in all the above sets. But for a general monopole these sets are all disjoint.

From now on we shall assume that the monopoles is general. Now we should like to show that

$$
H^{0}\left(S_{p}, L^{-z} I_{p}\left(2 m_{p}-1\right)\right)=m_{p}
$$

First we prove a vanishing result.

Proposition 17. $H^{0}\left(S_{p}, L^{-z} I_{p}\left(2 m_{p}-2\right)\right)=0$ for $z \in\left(\mu_{p+1}, \mu_{p}\right)$.

Proof. The proof is merely a matter of checking that the proof in [4] can be generalized to this case. Firstly we have the injection

$$
H^{0}\left(S_{p}, L^{-z} I_{p}\left(2 m_{p}-2\right)\right) \rightarrow H^{0}\left(S_{p}, L^{-z} E\left(2 m_{p}-2\right)\right),
$$

and the sequence

$$
H^{0}\left(\mathscr{T}, L^{-z} E\left(2 m_{p}-2\right)\right) \rightarrow H^{0}\left(S_{p}, L^{-z} E\left(2 m_{p}-2\right)\right) \stackrel{\delta}{\longrightarrow} H^{1}\left(\mathscr{T}, L^{-z} E(-2)\right),
$$

which is exact at the middle term. But using the fact from [4] that $H^{0}\left(\mathscr{T}, L^{\mu}(p)\right)=0$ 
if $\mu \neq 0$ for any $p$, and the filtration of $E(3)$, we have

$$
H^{0}\left(\mathscr{T}, L^{-z} E\left(2 m_{p}-2\right)\right)=0 \text { for } z \in\left(\mu_{p+1}, \mu_{p}\right) .
$$

So the composed map

$$
H^{0}\left(S_{p}, L^{-z} I_{p}\left(2 m_{p}-2\right)\right) \rightarrow H^{1}\left(\mathscr{T}, L^{-z} E(-2)\right)
$$

is injective. The latter space in (19) is, by the twistor correspondence [2] the space of all solutions $\varphi: \mathbf{R}^{3} \rightarrow \mathbf{C}^{n}$ of

$$
\left(d_{A}^{*} d_{A}+\Phi_{z}^{*} \Phi_{z}\right) \varphi=0 .
$$

If we can show that anything in the image of (19) is integrable on $\mathbf{R}^{3}$, then the vanishing theorem argument of [4] will show that it must be zero.

Because $I_{p}$ is contained in both $E_{p}^{+}$and $E_{n-p}^{-}$, we shall show that the class in the image of (19) can be represented by two forms

$$
\theta^{+} \in \Omega^{0,1}\left(\mathscr{T}, L^{-z} E_{p}^{+}(-2)\right), \quad \theta^{-} \in \Omega^{0,1}\left(\mathscr{T}, L^{-z} E_{n-p}^{-}(-2)\right)
$$

with $\theta^{+}-\theta^{-}=\bar{\partial} \gamma$ and $\theta^{+}, \theta^{-}, \gamma$, all having support in a compact neighbourhood of the spectral curve $S_{p}$.

If we pull everything back to $\hat{\theta}^{ \pm}, \hat{\gamma}$ on $\mathbf{R}^{3} \times S^{1}$, then these forms satisfy an equation like (2) on the fibres. If we choose $x$ outside of some ball of radius $R$, the intersection of the real section through $x$ with the compact neighbourhood of $S_{p}$ is two disjoint sets $V_{N}$ and $V_{S}$ which are neighbourhoods of $x /\|x\|$ and $-x /\|x\|$.

Because of (21) it follows [4] that for $\|x\| \geqq R$ there are constants $C$ and $\varepsilon \geqq 0$ such that

$$
\begin{aligned}
&\left\|\theta^{+}(x, u)\right\| \leqq C \exp (-\varepsilon x \cdot \mu) \quad u \in V_{N} \\
&\left\|\theta^{-}(x, u)\right\| \leqq C \exp (-\varepsilon|x \cdot \mu|) \quad u \in V_{S} .
\end{aligned}
$$

Write $\gamma=\gamma_{N}+\gamma_{S}$, where $\gamma_{N}=\gamma \mid V_{N}$ and $\gamma_{S}=\gamma \mid V_{S}$ and let $\theta=\theta^{+}-\bar{\partial} \gamma_{S}$. The form $\theta$ is cohomologous to $\theta^{+}$and satisfies $\theta\left|V_{N}=\theta^{+}, \theta\right| V_{S}=\theta^{-}$, so that

$$
\|\theta(x, \mu)\| \leqq \exp (-\varepsilon|x \cdot u|)
$$

for all $u$. Notice that $\theta$ is only well-defined for $\|x\| \geqq R$, but as the twistor correspondence can be applied between a neighbourhood of any point in $\mathbf{R}^{3}$ and a neighbourhood of the corresponding real section in $\mathscr{T}$, it follows that the function $\{x \mid\|x\| \geqq R\} \rightarrow \mathbf{C}$ defined by $\theta$ is the restriction of the function $\mathbf{R}^{3} \rightarrow \mathbf{C}$ defined by $\theta^{+}$. The function $\mathbf{R}^{3} \rightarrow \mathbf{C}$ therefore has the right decay properties to apply the argument in [4].

The forms $\theta^{+}, \theta^{-}$exist as in [4], but to get $\gamma$ having the correct support we have to be more careful. We can split the bundle $E$ smoothly as

$$
\begin{aligned}
E & =E_{p}^{+} \oplus W, \quad W \simeq E / E_{p}^{+}, \\
E^{\prime} & =W^{\prime} \oplus E_{n-p}^{-}, \quad W^{\prime} \simeq E / E_{n-p}^{-},
\end{aligned}
$$

with $\bar{\partial}$ operators $\left(\begin{array}{ll}\bar{\partial} & \theta \\ 0 & \bar{\partial}\end{array}\right)$ and $\left(\begin{array}{ll}\bar{\partial} & 0 \\ \theta^{\prime} & \bar{\partial}\end{array}\right)$. These are intertwined by a map $\left(\begin{array}{ll}A & B \\ C & D\end{array}\right)$, where we think of element of $E$ and $E^{\prime}$ as column vectors. 
If $\sigma \in H^{0}\left(S_{p}, L^{-z} I_{p}\left(2 m_{p}-2\right)\right)$ we can extend it smoothly in a neighbourhood of $S_{p}$ and then $\theta^{+}=\left(\bar{\partial} \sigma / \varphi_{p}, 0\right)^{t}$. Applying the map $\left(\begin{array}{ll}A & B \\ B & D\end{array}\right)$, we obtain

$$
\theta^{+}=\left(\begin{array}{ll}
A & B \\
C & D
\end{array}\right)\left(\begin{array}{l}
\bar{\partial} \sigma / \varphi_{p} \\
0
\end{array}\right)=\left(\begin{array}{c}
0 \\
C \bar{\partial}\left(\frac{\sigma}{\varphi_{p}}\right)-\theta^{\prime} \frac{(A \sigma)}{\varphi_{p}}
\end{array}\right)+\left(\begin{array}{cc}
\bar{\partial} & 0 \\
\theta^{\prime} & \bar{\partial}
\end{array}\right)\left(\begin{array}{c}
A \sigma / \varphi_{p} \\
0
\end{array}\right) .
$$

Now $A$ is the projection $E_{p}^{+} \rightarrow E / E_{n-p}^{-}$, hence $A \sigma$ vanishes on $S_{p}$ which has no multiple components, so $A \sigma / \varphi_{p}$ is well defined. Writing (24) as $\theta^{+}=\theta^{-}+\bar{\partial} \gamma$, we see that $\theta^{+}, \theta^{-}$, and $\gamma$ satisfy (21) and have the correct support.

Letting square brackets around a divisor denote the line bundle defined by that divisor we have

Lemma 25. $I_{p} \simeq L^{\mu_{p+1}}\left(-m_{p}-m_{p+1}\right) \otimes\left[S_{p+1, p}\right]$.

Proof. Consider the map $\hat{\xi}_{p}$ defined over $S_{p}$ by

$$
\begin{gathered}
\varphi_{p \nearrow} \Lambda^{p}\left(E / E_{n-p}^{-}\right) \\
0 \rightarrow \Lambda^{p} E_{p}^{+} \rightarrow \Lambda^{p} E_{p+1}^{+} \rightarrow \Lambda^{p-1} E_{p}^{+} \otimes E_{p+1}^{+} / E_{p}^{+} \rightarrow 0 .
\end{gathered}
$$

Using the definitions (15) we see that $\hat{\xi}_{p}$ is a surjection except on $S_{p+1, p}$. The identity $\Lambda^{p-1} E_{p}^{+} \simeq E_{p}^{+*} \otimes \operatorname{det}\left(E_{p}^{+}\right)$shows that $\hat{\xi}_{p}$ defines a map

$$
L^{\mu_{p+1}}\left(-m_{p}-m_{p+1}\right) \rightarrow E_{p}^{+}
$$

vanishing only on $S_{p+1, p}$ whose image is in $I_{p}$. In fact the map

$$
\Lambda^{p} E_{p}^{+} \otimes\left(E_{p+1}^{+} / E_{p}^{+}\right) \otimes \Lambda^{p}\left(E / E_{n-p}^{-}\right)^{*} \rightarrow E_{p}^{+}
$$

has the form

$$
y_{1} \wedge \cdots \wedge y_{p} \otimes\left(y_{p+1}+E_{p}^{+}\right) \otimes z \mapsto \sum_{i=1}^{p}(-1)^{i}<z,\left(y_{1}+E_{n-p}^{-}\right) \wedge \cdots\left(y_{i}+\hat{E}_{n-p}^{-}\right) \cdots \wedge y_{i},
$$

and if some $y_{i} \notin E_{n-p}^{-}$, then because $y_{i}, \ldots, y_{p}$ form a basis of $E_{p}^{+}$, some linear combination of $y_{i}, \ldots, y_{i-1}, y_{i+1}, \ldots, y_{p}$ is in $E_{n-p}^{-}$and the coefficient in front of $y_{i}$ vanishes. Hence we have a map $L^{\mu_{p+1}}\left(-m_{p}-m_{p+1}\right) \rightarrow I_{p}$ vanishing on $S_{p+1, p}$. It cannot vanish with multiplicity because we know that the induced map

$$
\Lambda^{p}\left(E / E_{n-p}^{-}\right)^{*} \rightarrow\left(\Lambda^{p-1} E_{p-1}^{+} \otimes E_{p+1}^{+} / E_{p}^{+}\right)^{*}
$$

has divisor $S_{p+1, p}+S_{p-1, p}[7]$. This completes the lemma.

From [7] we have that

$$
L^{\mu_{p+1}-\mu_{p}}\left(m_{p+1}+m_{p-1}\right)=\left[S_{p-1, p}\right] \otimes\left[S_{p+1, p}\right]
$$

as line bundles on $S_{p}$, and therefore

$$
L^{-\mu_{p}} I_{p}\left(m_{p}+l\right)=\left[S_{p-1, p}+2 S_{p, p+1}+\left(l-2 m_{p+1}-m_{p-1}\right) F \cap S_{p}\right],
$$

where $F$ is a fibre of $\mathscr{T} \rightarrow \mathbf{P}_{1}$ which avoids the singularity set of $S_{p}$. 
From Hartshorne [1] we have the Riemann-Roch Theorem for a possibly singular curve

$$
\operatorname{dim} H^{0}\left(S_{p},[D]\right)-\operatorname{dim} H^{1}\left(S_{p},[D]\right)=|D|+\text { constant, }
$$

where $D$ avoids the singularity set of $S_{p}$. Letting $D=S_{p} \cap F$, and applying Riemann-Roch for compatified twistor space as in [4], we calculate the constant as $1-\left(m_{p}-1\right)^{2}$. So

$$
\operatorname{dim} H^{0}\left(S_{p}-L^{-\mu_{p}} I_{p}\left(m_{p}+l\right)\right)-\operatorname{dim} H^{1}\left(S_{p}, L^{-\mu_{p}} I_{p}\left(m_{p}+l\right)\right)=m_{p}\left(l-m_{p}\right)+2 m_{p} .
$$

The argument of [4] can now be applied to give

$$
\begin{array}{ll}
H^{0}\left(S_{p}, L^{-z} I_{p}\left(2 m_{p}+l\right)\right)=0, & l \leqq m_{p}-2 \\
H^{1}\left(S_{p}, L^{-z} I_{p}\left(2 m_{p}+l\right)\right)=0, & l \geqq m_{p}-2,
\end{array}
$$

and $z \in\left(\mu_{p+1}, \mu_{p}\right)$.

In particular we have now proved that

$$
\operatorname{dim} H^{0}\left(S_{p}, L^{-z} I_{p}\left(2 m_{p}-1\right)\right)=m_{p}
$$

for $z \in\left(\mu_{p+1}, \mu_{p}\right)$. So let $V_{z}=H^{0}\left(S_{p}, L^{-z} I_{p}\left(2 m_{p}-1\right)\right)$, and following the argument of [4] we have the multiplication map

$$
H^{0}\left(S_{p}, \mathcal{O}(2)\right) \otimes V_{z} \rightarrow H^{0}\left(S_{p}, L^{-z} I_{p}\left(2 m_{p}+1\right)\right)
$$

with kernel $K_{z}$ and

Proposition 29. The map $K_{z} \rightarrow V_{z}$ defined by

$$
\eta \otimes s_{0}+i \sum_{j=1}^{3} z_{j} \otimes s_{j} \mapsto s_{0}
$$

is an isomorphism, therefore there exist endomorphisms $H_{i}(z)$, so that $K_{z}$ is the span of

$$
\eta \otimes s_{0}+i \sum_{j=1}^{3} z_{j} \otimes H_{j}(z) s_{0}
$$

and the spectral curve $S_{p}$ is the divisor of

$$
\operatorname{det}\left(\eta 1+i \sum_{j=1}^{3} z_{j} H_{j}(z)\right) .
$$

Proof. The proof that $K_{z} \rightarrow V_{z}$ is an isomorphism is the same as in [4]. Then $\operatorname{det}\left(\eta 1+i \sum_{j=1}^{3} z_{j} H_{j}(z)\right)$ defines a curve of the same degree as $S_{p}$, and this determinant clearly vanishes on $S_{p}$, so as $S_{p}$ has no multiple components this curve must be $S_{p}$.

\section{The Identity of the Curves}

To show that curves $S_{i}$ and $N_{i}$ are the same it now suffices to find an isomorphism $V_{z} \rightarrow W_{z}$ which intertwines the action of $H_{i}(z)$ and $T_{i}(z)$. It would, in fact, be enough to do this for some $z \in\left(\mu_{p+1}, \mu_{p}\right)$, but it will follow from the method of proof that it can be done for any $z \in\left(\mu_{p+1}, \mu_{p}\right)$. 
The isomorphism follows from the twistor correspondence between solutions of the Dirac equation and a sheaf cohomology group, described in four dimensions in [2].

Proposition 30. The twistor correspondence gives an isomorphism from $V_{z}$ to the space $W_{z}$ of $L^{2}$ solutions of

$$
\left(D_{A}+\Phi_{z}\right) s=0
$$

Proof. Again as in Proposition 17 we use a coboundary to inject $V_{z}$ into $H^{1}\left(\mathscr{T}, L^{-z} E(-1)\right)$, and from the general twistor correspondence (see [2] for the four dimensional case which can be readily adapted) these cohomology classes are in bijective correspondence with the solutions of (31). Because $\operatorname{dim} V_{z}=\operatorname{dim} W_{z}$, it suffices to show that the image of $V_{z}$ under these two maps corresponds to integrable solutions of (31).

First let us review the twistor correspondence and for simplicity ignore the bundle $E$. Let $\theta$, then represent a cohomology class in $H^{1}(\mathscr{T}, \mathcal{O}(-1))$ and pull $\theta$ back to $\hat{\theta}$ on $\mathbf{C}^{3} \times \mathbf{P}_{1}$, which fibres over $\mathscr{F}$ via the map $\left(x^{i}, \zeta\right) \mapsto\left(\sum_{i=1}^{3} x^{i} z^{i}(\zeta),(\zeta)\right)$, (see [3]). The vertical tangent vectors to the fibres of

$$
\mathbf{C}^{3} \times \mathbf{P}_{1} \rightarrow \mathscr{T}
$$

form a sub-bundle $T_{v}$ of the trivial bundle $\mathbf{C}^{3}$ on each $\mathbf{P}_{1}$, and we have

$$
0 \rightarrow T_{v} \rightarrow \mathbf{C}^{3} \rightarrow \mathcal{O}(2) \rightarrow 0 .
$$

There is a vertical derivative $d_{v}$ on $\mathbf{C}^{3} \times \mathbf{P}_{1}$ obtained by projecting from $\mathbf{C}^{3}$ to $T_{v}^{*}$, and we have $d_{v} \theta=0$ because it is pulled back from $\mathscr{T}$. If we think of $\mathbf{P}_{1}$ as the quadric of all null lines in $\mathbf{C P}_{2}$, then the fibre of $T_{v}$ is the orthogonal plane to each null line. Because the lines are null they are contained in their orthogonal plane, and we have the exact sequence

$$
0 \rightarrow \mathcal{O}(-2) \rightarrow T_{v} \rightarrow \mathbf{C} \rightarrow 0 .
$$

Now $H^{1}\left(\mathbf{P}_{1}, \mathcal{O}(-1)\right)=0$, so for each form $\theta$ we can find an $s(x, \zeta) \in \Gamma\left(\mathbf{C}^{3} \times\right.$ $\left.\mathbf{P}_{1}, \mathcal{O}(-1)\right)$ with $\theta-\bar{\partial}_{\mathbf{P}_{1}} s=0$ on $\mathbf{P}_{1}$. The $s(x, \zeta)$ is unique because $H^{0}\left(\mathbf{P}_{1}, \mathcal{O}(-1)\right)=0$ and is smooth in $x$ because it satisfies an elliptic partial differential equation with smooth coefficients. We shall see later that we can construct $s$ quite explicitly.

If $p: \mathbf{C}^{3} \rightarrow T_{v}^{*}$ is the projection, we have $d_{v}=\sum_{i=1}^{3} p\left(e_{i}\right)\left(\partial / \partial x_{i}\right)$, where each $p\left(e_{i}\right)$ is holomorphic, so $\left[d_{v}, \bar{\partial}_{\mathbf{P}_{1}}\right]=0$. It follows then that $\bar{\partial}_{\mathbf{P}_{1}} d_{v} s=d_{v} \bar{\partial}_{\mathbf{P}_{1}} s=d_{v} \theta=0$, and therefore $d_{v} s$ takes values in $H^{0}\left(\mathbf{P}_{1}, T_{v}^{*}(-1)\right)$. From the exact sequence (32) $H^{0}\left(\mathbf{P}_{1}, T_{v}^{*}(-1)\right) \rightleftharpoons H^{0}\left(\mathbf{P}_{1}, \mathcal{O}(1)\right) \simeq \mathbf{C}^{2}$, and this defines the Dirac field. Under these identifications it can be checked that $d_{v} s=\sum_{i=1}^{3} z_{i}\left(\partial s / \partial x_{i}\right)$. Note that $d_{v} s$ depends only on the class of $\theta$. In fact if $\theta^{\prime}=\theta+\bar{\partial} \mu$, then $p^{*} \theta^{\prime}=p^{*} \theta+\bar{\partial} p^{*} \mu$. By the uniqueness of the solutions of $p^{*} \theta^{\prime}=\bar{\partial} s^{\prime}$, we have $s^{\prime}=s+p^{*} \mu$. Hence $d_{v} s^{\prime}=d_{v} s+$ $d_{v} p^{*} \mu=d_{v} s+0$.

We can proceed now as in the proof of Proposition (17) and show that a class 
in the image of $V_{z}$ inside $H^{1}\left(\mathscr{T}, L^{-z} E(-1)\right)$ can be represented by two forms $\theta^{+}$ and $\theta^{-}$with $\theta^{+}=\theta^{-}-\bar{\partial} \gamma, \theta^{+}, \theta^{-}$and $\gamma$, all having support in a compact neighbourhood of the spectral curve and

$$
\theta^{+} \in \Omega^{0,1}\left(L^{-z} E_{p}^{+}(-1)\right), \quad \theta^{-} \in \Omega^{0,1}\left(L^{-z} E_{n-p}^{-}(-1)\right) .
$$

These forms can be lifted to $\mathbf{R}^{3} \times S^{2} \rightarrow \mathbf{C}^{3} \times \mathbf{P}_{1}$ and identified with forms $\hat{\theta}^{+}, \hat{\theta}^{-}$ taking values in $\mathbf{C}^{n} \otimes \mathcal{O}(-1)$ satisfying the differential equation $\nabla_{\gamma}-i \Phi_{z}$ along the fibres of $\mathbf{R}^{3} \times \mathbf{P}_{1} \rightarrow \mathscr{T}$. Choose an $R>0$ so that the intersection of the compact neighbourhood of $S_{p}$ with any real section for an $x$ with $\|x\| \geqq R$ consists of two disjoint sets $V_{N}$ and $V_{S}$ which are neighbourhoods of $x /\|x\|$ and $-x /\|x\|$. From the standard theory of the asymptotic behaviour of solutions of $\nabla_{\gamma}-i \phi_{z}$ (see [3]) we have

$$
\left\|\hat{\theta}^{+}\right\|<C e^{-\varepsilon|x \cdot \mu|} \quad \text { on } \quad V_{N}, \quad\left\|\hat{\theta}^{-}\right\|<C e^{-\varepsilon|x \cdot \mu|} \quad \text { on } \quad V_{S}
$$

for some $\varepsilon>0$ and $C$ fixed. Let us now work only on the set $\{x \mid\|x\| \geqq R\} \times \mathbf{P}_{1}$. Then letting $\gamma=\gamma_{N}+\gamma_{S}$, where $\gamma_{N}, \gamma_{S}$ have support on $V_{N}$ and $V_{S}$, we can define $\theta=\theta^{+}-\bar{\partial} \gamma_{S}$ so on $V_{N}, \theta=\theta^{+}$and on $V_{S}, \theta=\theta^{+}-\bar{\partial} \gamma_{s}=\left(\theta^{+}-\partial \gamma\right) \mid V_{s}=\theta^{-}$. Because $\theta$ is cohomologous to $\theta^{+}$it will induce the same Dirac field on $\{x \mid\|x\| \geqq R\}$ and we have that on

and on

$$
V_{N}, \quad\|\theta\|<C e^{-\varepsilon|x \cdot \mu|},
$$

$$
V_{S}, \quad\|\theta\|<C e^{-\varepsilon|x \cdot \mu|} .
$$

To obtain the Dirac field we want to solve

$$
\bar{\partial}_{\mathbf{P}_{1}} s(x)=\theta(x) \text {. }
$$

This can be done explicitly using the Cauchy kernel. Recall that if $f(\xi)$ has compact support in $\mathbf{C}$ then

$$
(K f)(\mu)=\frac{1}{2 \pi i} \int f(\xi) \frac{1}{\xi-\mu} d \xi \wedge d \bar{\xi}
$$

is smooth and satisfies $(\partial / \partial \bar{\mu}) K f=f$.

The Cauchy kernel $K$ has, however, a coordinate-free interpretation on $\mathbf{P}^{1}$, if we use the line bundle $\mathcal{O}(-1)$. Since the cotangent bundle of $\mathbf{P}^{1}$ in $\mathcal{O}(-2)$, which has with respect to a local affine coordinate $\xi$ a trivialization $d \xi$, then a local trivialization of $\mathcal{O}(-1)$ is given by $d \xi^{1 / 2}$. Now if we change coordinates by $\xi=(a \xi+b) /(c \xi+d)$ and $\tilde{\mu}=(a \mu+b) /(c \mu+d)$ with $a d-b c=1$, then

$$
\frac{d \tilde{\xi}^{1 / 2} d \tilde{\mu}^{1 / 2}}{(\tilde{\xi}-\tilde{\mu})}=\frac{d \xi^{1 / 2} d \mu^{1 / 2}}{(c \xi+d)(c \mu+d)}\left\{\frac{1}{\left(\frac{a \xi+b}{c \xi+d}\right)-\left(\frac{a \mu+b}{c \mu+d}\right)}\right\}=\frac{d \xi^{1 / 2} d \mu^{1 / 2}}{(\xi-\mu)}
$$

Then if $\theta$ is a $(0,1)$ form with values on $\mathcal{O}(-1)$, we may write $\theta=f(\xi) d \xi^{1 / 2} d \bar{\xi}$ locally and

$$
S=\frac{1}{2 \pi_{i}} \int f(\xi) d \xi^{1 / 2} \cdot \frac{d \xi^{1 / 2} d \mu^{1 / 2}}{(\xi-\mu)}=\frac{d \mu^{1 / 2}}{2 \pi_{i}} \int f(\xi) \cdot \frac{1}{\xi-\mu} d \xi d \bar{\xi}
$$


is a well-defined global section of $\mathcal{O}(-1)$ satisfying $\bar{\partial} s=\theta$. What we want to estimate now is the asymptotic behaviour of $d_{V} s$, but this is

$$
d_{V} s=\int \nabla_{i} \theta(x, \xi) \frac{z_{1}(\eta)}{\eta-\xi} d \xi \wedge d \bar{\xi}
$$

and as in [3], if a solution of $\nabla_{\gamma}-i \Phi_{z}$ decays, so do its derivatives, so this gives us control over the asymptotic decay of $d_{V} s$ which is our Dirac field.

Because the Dirac field decays exponentially it is integrable as required.

We have seen that under the twistor correspondence the space $V_{z}=$ $H^{0}\left(S_{p}, L^{-z} I_{p}\left(2 m_{p}-1\right)\right)$ is isomorphic to the space $W_{z}$ of integrable solutions to $\left(D_{A}+\Phi_{z}\right) \Psi=0$. Now consider the map

$$
H^{0}(\mathscr{T}, \mathcal{O}(2)) \otimes V_{z} \stackrel{m}{\rightarrow} H^{0}\left(S_{p}, L^{-z} I_{p}\left(2 m_{p}+1\right)\right)
$$

which was used to define the $H_{i}$. The space $H^{0}\left(S_{p}, L^{-z} I_{p}\left(2 m_{p}-1\right)\right)$ can be mapped by a coboundary to $H^{1}\left(\mathscr{T}, L^{-z} E(1)\right)$. Consider a $(0,1)$ form $w$ representing a class in $H^{1}\left(\mathscr{T}, L^{-z} E(1)\right)$. This can be pulled back to $\mathbf{C}^{3} \times \mathbf{P}_{1}$ to give $\hat{w}$. Just as with $H^{1}\left(\mathscr{T}, L^{-z} E(-1)\right)$ we can solve $\bar{\partial}_{\mathbf{P}_{1}} s=w$, but now $s$ is not unique but can be changed by adding elements of $H^{0}\left(\mathbf{P}_{1}, L^{-z} E(1)\right)$. Letting $\Psi=d_{V} s: \mathbf{C}^{3} \rightarrow H^{0}$ $\left(\mathbf{P}_{1}, T_{v}^{*}(1)\right) \otimes \mathbf{C}^{n} \simeq S^{1} \otimes S^{2} \otimes \mathbf{C}^{n}$, where $S^{k}$ is the irreducible representation of $S U(2)$ of dimension $k$, it can be checked that $\Psi$ is in the $H^{1}$ of the elliptic complex

$$
C^{\infty}\left(\mathbf{C}^{3}, S^{1} \otimes \mathbf{C}^{n}\right) \stackrel{T}{\longrightarrow} C^{\infty}\left(\mathbf{C}^{3}, S^{1} \otimes S^{2} \mathbf{C}^{n}\right) \stackrel{D}{\longrightarrow} C^{\infty}\left(\mathbf{C}, S^{3} \otimes \mathbf{C}^{n}\right)
$$

(see [2] for the four-dimensional version of this). If we let $e_{1}, e_{2}, e_{3}$ be a basis for $\mathbf{C}^{3} \simeq \operatorname{sl}(2, \mathbf{C})$ with $e_{j}^{2}=-1, e_{1} e_{2}=e_{3}$, etc., then we have that $T$ is the twistor operator

and

$$
T(\Psi)=-\frac{1}{2} \sum_{j=1}^{3}\left(D_{A}-\Phi_{z}\right)\left(e_{j} \Psi\right) \otimes e_{j}
$$

$$
D\left(\sum_{i=1}^{3} \varphi_{i} \otimes \sigma^{i}\right)=\operatorname{sym} \sum_{i=1}^{3}\left\{\left(D_{A}-\Psi_{z}\right) \varphi_{i} \otimes e_{i}\right\},
$$

where sym: $S^{2} \otimes S^{1} \rightarrow S^{3}$ is the symmetrization map $S^{2}\left(\mathbf{C}^{2}\right) \otimes \mathbf{C}^{2} \rightarrow S^{3}\left(\mathbf{C}^{2}\right)$.

If $\eta, z_{i} \in H^{0}(\mathscr{T}, 0(2))$ it can be readily checked that the multiplication map is

$$
\begin{aligned}
m: \mathbf{C}^{4} \otimes W_{z} & \rightarrow C^{\infty}\left(\mathbf{R}^{3}, S^{1} \otimes S^{2}\right), \\
\eta \otimes \varphi_{0}+i \sum_{j=1}^{3} z_{j} \otimes \varphi_{j} & \mapsto \sum_{j=1}^{3} x^{i} \varphi_{0} \otimes e_{j}+\sum_{j=1}^{3} i \varphi_{j} \otimes e_{j} .
\end{aligned}
$$

Finally we have

Proposition 35. The element $\eta \otimes \varphi_{0}+i \sum_{j=1}^{3} z_{j} \otimes \varphi_{j}$ is in the kernel of $m$ if there is some $\chi: \mathbf{R}^{3} \rightarrow S^{1}$ with

$$
\sum_{j=1}^{3}\left(x^{i} \varphi_{0}+i \varphi_{j}\right) \otimes e_{j}=\frac{-1}{2} \sum_{j=1}^{3}\left(D_{A}-\Phi_{z}\right) e_{j} \chi \otimes e_{j}
$$


or

$$
x^{i} \varphi_{0}+i \varphi_{j}=-\frac{1}{2}\left(D_{A}-\Phi_{z}\right) e_{j} \chi \quad i=1,2,3 .
$$

Now let us assume that $\chi$ is integrable. Then $D_{A}-\Phi_{z}$ is the adjoint of $D_{A}+\Phi_{z}$, so if $\pi$ is the projection $L^{2}\left(\mathbf{R}^{3}, S^{1}\right) \rightarrow\left\{\right.$ kernel of $\left.D_{A}+\Phi_{z}\right\}=W_{z}$, applying $\pi$ to Eq. (36) gives

$$
i \pi\left(x^{j} \varphi_{0}\right)=\varphi_{j} .
$$

Comparing with (6) this means the kernel of the multiplication map is all $\eta \otimes \varphi_{0}+i \sum_{i=1}^{3} z^{i} \otimes T_{i}(z) \varphi_{0}$ and we therefore have

Proposition 37. The isomorphisms $V_{z} \rightarrow W_{z}$ intertwines the endomorphisms $T_{i}(z)$ and $H_{i}(z)$, and hence Nahm's curves $N_{i}$ and the spectral curves coincide for a general monopole.

Proof. It remains to show that $\chi$ is integrable. Return to Proposition 17 and 30 and let $\theta_{j}^{ \pm}, j=0,1,2,3$ be the representative form for the class isomorphic to $\varphi_{j}$ as used in those proofs. Then from the construction of $\varphi_{j}^{ \pm}$there are $\mu^{ \pm}$having support in the compact neighbourhood of $S_{p}$ and satisfying

$$
\begin{gathered}
\mu^{+} \in \Omega^{0}\left(\mathscr{T}, L^{-z} E_{p}^{+}(-1)\right), \\
\mu^{-} \in \Omega^{0}\left(\mathscr{T}, L^{-z} E_{n-p}^{-}(-1)\right), \\
\eta \theta_{0}^{ \pm}-i \sum_{j=1}^{3} z_{j} \theta_{j}^{ \pm}=\bar{\partial} \mu^{ \pm},
\end{gathered}
$$

and

$$
\mu^{+}=\mu^{-}+\gamma
$$

When we form $\theta=\theta^{+}-\left.\overline{\partial \gamma}\right|_{V_{s}}$, we can also form $\mu=\mu^{+}-\left.\gamma\right|_{V_{s}}$ and we have

$$
\eta \theta_{0}-i \sum_{j=1}^{3} z_{j} \theta_{j}=\mu .
$$

Pulling back to $\mathbf{R}^{3} \times \mathbf{P}_{1}$ gives

$$
\eta \hat{\theta}_{0}-i \sum_{j=1}^{3} z_{j} \hat{\theta}_{j}=\hat{\mu}
$$

and if $\theta_{j}=\bar{\partial} s_{j}, j=0,1,2,3$, then

$$
\eta s_{0}-i \sum_{j=1}^{3} z_{j} s_{j}=\hat{\mu}+\chi
$$

is the equation defining $\chi: \mathbf{R}^{3} \rightarrow H^{0}\left(\mathbf{R}_{1}, L^{-z} \widehat{E}(-1)\right)$.

Using the Cauchy kernel argument we can obtain decay estimates on the $s_{j}$, and because the $\mu$ has the same support properties and takes its values in the same sub-bundles as $\theta$ we can also obtain decay estimates on $\hat{\mu}$. It follows from (38) that $\chi$ must decay exponentially at infinity and therefore is integrable. 


\section{Conclusion}

We have seen that Nahm's curves are the same as the spectral curves of a general monopole. In fact a lot more is true, namely that any collection of spectral data satisfying the vanishing conditions in Proposition 17 arise from a monopole. These results will appear in [5].

The ADHM construction and therefore Nahm's construction really only work for $S U(n)$ (and hence the other classical groups with suitable modifications). However the spectral curves are defined for the exceptional groups also and it would be interesting to know if, as has been suggested by Atiyah, one can perhaps solve Nahm's equations on the "dual" of the Dynkin diagram for a general monopole.

Acknowledgements. The second author, M. K. Murray, would like to thank A. Carey, R. Moore and R. Richardson for useful discussions.

\section{References}

1. Hartshorne, R.: Algebraic geometry. New York, Heidelberg, Berlin: Springer 1977

2. Hitchin, N. J.: Linear field equations on self-dual spaces. Proc. R. Soc. Lond. A370, 173-191 (1980)

3. Hitchin, N. J.: Monopoles and geodesics. Commun. Math. Phys. 83, 579-602 (1982)

4. Hitchin, N. J.: On the construction of monopoles. Commun. Math. Phys. 89, 145-190 (1983)

5. Hurtubise, J. C., Murray, M. K.: On the construction of $S U(n)$ monopoles (to appear)

6. Murray, M. K.: Monopoles and spectral curves for arbitrary Lie groups. Commun. Math. Phys. 90, 263-271 (1983)

7. Murray, M. K.: Non-abelian magnetic monopoles. Commun. Math. Phys. 96, 539-565 (1984)

8. Nahm, W.: The algebraic geometry of multimonopoles. Bonn University preprint, BONN-HE-82-30

Communicated by A. Jaffe

Received July 23, 1987 